\title{
General Relativity Theory Violates the Energy Conservation Law, which is the Fundamental Law of Physics Including the Curved Space-Time Metric
}

\author{
Jaroslav Hynecek ${ }^{1}$ \\ ${ }^{1}$ Isetex, Inc., 905 Pampa Drive, Allen, TX 75013, USA \\ Correspondence: Jaroslav Hynecek, Isetex, Inc., 905 Pampa Drive, Allen, TX 75013, USA. E-mail: \\ jhynecek@netscape.net
}

Received: May 15, 2018

Accepted: July 24, 2018

Online Published: July 26, 2018

doi:10.5539/apr.v10n4p87

URL: https://doi.org/10.5539/apr.v10n4p87

\begin{abstract}
The violation of energy conservation law is a death sentence for the General Relativity Theory (GRT). This paper investigates the correctness of the General Relativity Theory by studying the energy conservation during the relativistic free fall of a small test body in a uniform gravitational field. The paper compares predictions of energy conservation obtained from the GRT and from the Metric Theory of Gravity (MTG). It is found that the gravitational mass dependence on velocity in the GRT is not correct, because this dependency leads to a prediction of violation of energy conservation while the MTG having a different gravitational mass dependency on velocity predicts correctly the energy conservation.
\end{abstract}

\section{Introduction}

The theories describing the free fall motion in a uniform gravitational field of a test body are well understood in both; the GRT and the MTG. In the GRT the inertial mass and the gravitational mass are assumed identical with identical dependencies on velocity. In the MTG, on the other hand, the gravitational mass depends on velocity differently than the inertial mass (Hynecek, 2005, 2017). It is thus simple for both theories to derive equations describing the free fall velocity and from that the energy of a test body that falls in a uniform gravitational field that also possesses the curved space-time metric.

\section{Theories}

It is well known that the gravitational field distorts the space-time. It is thus necessary to use the metric describing this distortion. However, the metric that is used for the analysis was not derived from the GRT for the uniform gravitational field, but was derived and is described in the previous publication (Hynecek, 2009a) with its differential metric line element as follows:

$$
d s^{2}=\frac{(c d t)^{2}}{\left(1-g \cdot z / c^{2}\right)^{2}}-\frac{d z^{2}}{\left(1-g \cdot z / c^{2}\right)^{2}}
$$

Here it was considered for the sake of simplicity that the motion occurs only in the negative $z$ direction $z \leq 0$ with the uniform gravitational acceleration equal to a constant $g$. It is also worth noticing that the speed of light in the $z$ direction in this space-time is not affected by the gravitational field and retains its vacuum value $c$. For a reader's convenience a simplified version of this metric derivation is given in the appendix.

The motion of a small test body in this uniform gravitational field will be analyzed using the well-known and ages tested Lagrange formalism. The Lagrangian characterizing the free fall in this curved space-time is therefore as follows:

$$
L=\frac{(c d t / d \tau)^{2}}{\left(1-g \cdot z / c^{2}\right)^{2}}-\frac{(d z / d \tau)^{2}}{\left(1-g \cdot z / c^{2}\right)^{2}}
$$

The corresponding Euler-Lagrange equations of motion are then readily found following the formalism: 


$$
\frac{d}{d \tau}\left(\frac{\partial L}{\partial(d z / d \tau)}\right)=\frac{\partial L}{\partial z}
$$

First for the time variable the result is:

$$
\frac{d t}{d \tau}=\left(1-g \cdot z / c^{2}\right)^{2}
$$

where the constant of integration was suitably selected such that at the origin $z=0$ the $d t=d \tau$. For the space variable the computation is slightly more complicated with the first step as follows:

$$
-\frac{d}{d \tau}\left(\frac{(d z / d t)(d t / d \tau)}{\left(1-g \cdot z / c^{2}\right)^{2}}\right)=\frac{(d t / d \tau)^{2} g}{\left(1-g \cdot z / c^{2}\right)^{3}}-\frac{(d z / d \tau)^{2} g / c^{2}}{\left(1-g \cdot z / c^{2}\right)^{3}}
$$

This formula can be simplified using Equation 4 and the definition of velocity $v=d z / d t$ as follows:

$$
\frac{d v}{d t}=-\frac{g\left(1-v^{2} / c^{2}\right)}{\left(1-g \cdot z / c^{2}\right)}
$$

The Lagrangian itself is also the first integral $\left(L=c^{2}\right)$ and this leads to the following relation:

$$
\frac{1}{\left(1-g \cdot z / c^{2}\right)^{2}}=1-\frac{v^{2}}{c^{2}}
$$

It is now easy to verify that the energy is conserved during the fall. Using Equation 7, taking the square root of the formula, multiplying the result by $c^{2}$ and by the rest mass of the falling body $m_{0}$, we can write:

$$
\frac{m_{0} c^{2}}{\sqrt{1-v^{2} / c^{2}}}=m_{0} c^{2}-m_{0} g \cdot z
$$

This clearly indicates that relativistic mass-energy is properly and exactly conserved $(z \leq 0)$ during the fall as expected, thus satisfying the energy conservation rule. This result also confirms that the metric introduced in Equation 1 is a correct metric for the uniform gravitational field space-time.

In the next steps we will find equation for the force that the uniform gravitational field exerts on the falling body. This is important for a comparison of MTG and GRT theories. Using Equation 7 in Equation 6 the formula for acceleration as a function of velocity can be found:

$$
\frac{d v}{d t}=-g\left(1-v^{2} / c^{2}\right)^{\frac{3}{2}}
$$

This equation can be rearranged and multiplied by the rest mass of the falling body with the result:

$$
\frac{d}{d t}\left(\frac{m_{0} v}{\sqrt{1-v^{2} / c^{2}}}\right)=-m_{0} g
$$

This formula is the relativistic forces balance equation where the left hand side is the inertial force formula and the right hand side is the gravitational force formula. The force that is acting on the falling body is thus clearly constant and independent of velocity.

However, in the previous publications (Hynecek, 2005, 2009) it was claimed that the gravitational mass in MTG depends on velocity differently than the inertial mass in contrast to the GRT dependency. This apparent discrepancy needs to be now reconciled.

From the general contravariant expression for the gravitational force vector acting in a curved space-time that was introduced in previous publication (Hynecek, 2009a) the $z$ component can be written as:

$$
F_{g}=-g^{z z} \frac{\partial \varphi}{\partial z} m_{0} \sqrt{g_{t t}} \sqrt{1-v^{2} / c^{2}}
$$


where $\varphi$ is the gravitational potential. Using the metric coefficients introduced in the differential metric line element the gravitational force acting on the test body is thus evaluated with the help of Equation 7 to be:

$$
F_{g}=-\frac{\left(1-g \cdot z / c^{2}\right)^{2} g \cdot m_{0}}{\left(1-g \cdot z / c^{2}\right)\left(1-g \cdot z / c^{2}\right)}=-m_{0} g
$$

This is very interesting result. While the gravitational mass changes with velocity differently than the inertial mass:

$$
m_{g}=m_{0} \sqrt{1-v^{2} / c^{2}} \text {, the force on the falling test body stays constant. This is the effect of a curved space-time. }
$$

This result now provides the tool to investigate the GRT mass dependence on velocity and consequently its effect on the conservation of energy during the fall. This result also justifies the calculation of energy when slowly lifting the test body by the distance $z$ as is being acted upon by this constant force. The gained potential energy is calculated simply as follows:

$$
E_{g}=m_{0} g \cdot|z|
$$

Using now the same approach for the GRT force as was used above for the MTG force it is clear that by modifying Equation 12 for the GRT gravitational mass dependence on velocity the gravitational force on the test body according to GRT is:

$$
F_{g r t}=-g^{z z} \frac{\partial \varphi}{\partial z} m_{0} \sqrt{g_{t t}} \frac{\sqrt{1-v^{2} / c^{2}}}{\left(1-v^{2} / c^{2}\right)}
$$

This results in the following GRT forces balance equation:

$$
\frac{d}{d t}\left[\frac{m_{0} v}{\sqrt{1-v^{2} / c^{2}}}\right]=-g \frac{m_{0}}{\left(1-v^{2} / c^{2}\right)}
$$

The formula in Equation 15 can be rearranged and simplified resulting in the relation for the small test body acceleration as is valid in the GRT:

$$
\frac{d v}{d t}=-g \sqrt{1-v^{2} / c^{2}}
$$

In the next step it is necessary to rearrange this relation as a function of distance $z$. This can be accomplished as follows:

$$
\frac{d v}{\sqrt{1-v^{2} / c^{2}}}=-g \frac{d t}{d z} d z
$$

After integration the result is:

$$
\sqrt{1-v^{2} / c^{2}}=1-g \cdot|z| / c^{2}
$$

The energy difference will be calculated by comparing the potential energy that is exerted by lifting the test body very slowly in the uniform gravitational field $g$ by a distance $z$ to the energy that is gained when the body falls back the same distance $z \leq 0$.

$$
\Delta E=\left(m_{0} c^{2}+m_{0} g \cdot|z|\right)-\frac{m_{0} c^{2}}{\sqrt{1-v^{2} / c^{2}}}=\left(m_{0} c^{2}+m_{0} g \cdot|z|\right)-m_{0} c^{2}\left(1+\frac{g \cdot|z|}{c^{2}}+\left(\frac{g \cdot z}{c^{2}}\right)^{2}+\cdots\right)
$$

By expanding the result into a power series as is shown in Equation 19 and neglecting the higher order terms the energy difference becomes as follows: 


$$
\Delta E \cong-\frac{m_{0} c^{2}}{4}\left(\frac{2 g \cdot z}{c^{2}}\right)^{2} \cong-\frac{m_{0} c^{2}}{4}\left(\frac{v^{2}}{c^{2}}\right)^{2}
$$

This is a very strange result. It seems that the falling body is gaining some additional energy from an unknown source on top of the energy that is predicted from the standard relativistic energy formula:

$$
E=\frac{m_{0} c^{2}}{\sqrt{1-v^{2} / c^{2}}}
$$

This is not reasonable and it is pointing to a problem that exists in the GRT for a long time. The gravitational mass cannot depend on velocity the same way as the inertial mass. The conservation of energy in GRT is thus violated. The GRT is, therefore, not a valid theory of gravity.

For a better understanding of the amount of energy violation a graph is shown below in FIG.1.

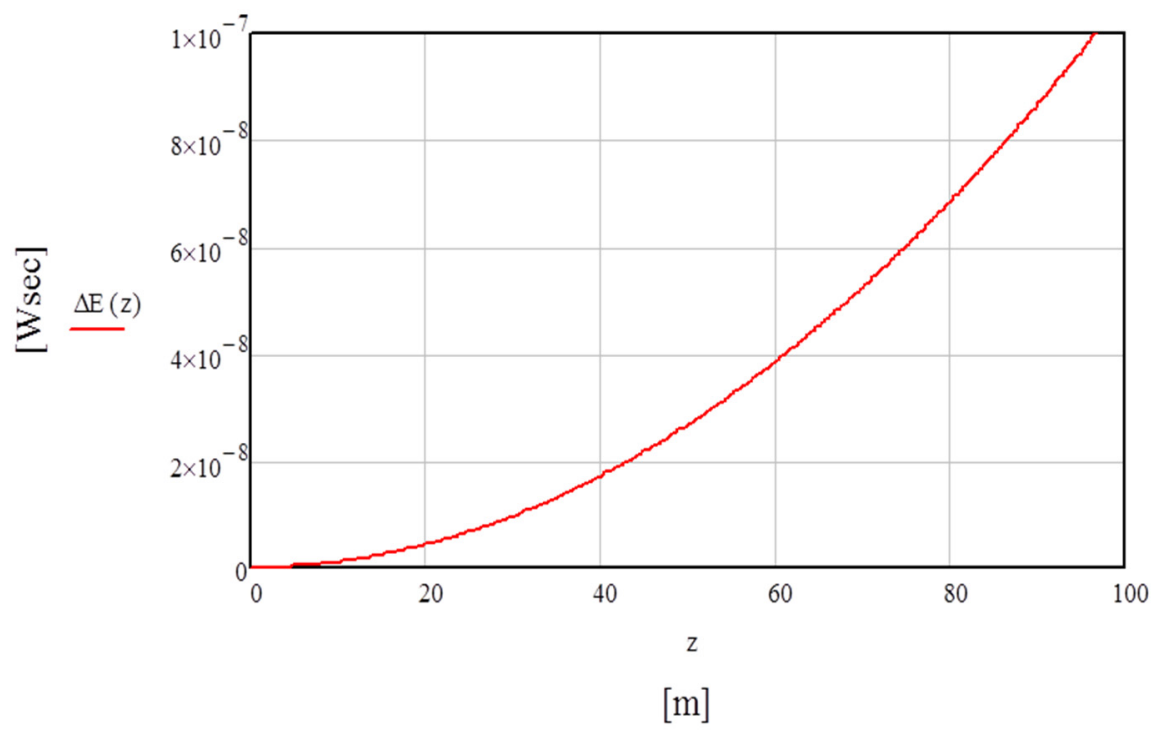

Figure 1. the violation of energy conservation law in dependence on a distance of fall $z$ for a mass of $10,000 \mathrm{~kg}$ and the Earth's gravitational acceleration $g=9.80665 \mathrm{~m} / \mathrm{s}^{2}$ according to GRT

\section{Conclusions}

The paper derived simple expression for the energy conservation during the small test body free fall in a uniform gravitational field that also included the effect of gravity on the curvature of space-time. It was shown that the energy conservation derived according to the GRT mass dependence on velocity is violated. This is unacceptable and this fact proves the invalidity of GRT. The paper thus clearly verified that this problem has its root cause in the identical dependency of inertial mass and gravitational mass on velocity in GRT.

Fortunately the new MTG theory of gravity was recently developed where the dependency of gravitational mass on velocity is different than in the GRT. This solved the energy conservation problem.

The presented results have fatal consequences for the GRT, because unquestionably prove its incorrectness. These findings thus have a significant impact on all the theories based on the GRT such as the Big Bang and similar ridiculous models of the Universe.

The author hopes that the main stream relativists finally recognize this problem and abandon the GRT with all its ridiculous claims of existence of Black Holes, Event Horizons, and the Big Bang Universe with its accelerating expansion to infinity from nothing. 


\section{References}

Hynecek, J. (2017). Review of Internal Inconsistencies in GRT and Comparisons to MTG. The General Science Journal. Retrieved from http://gsjournal.net/Science-Journals/Research\%20Papers/View/7070

Hynecek, J. (2005). Remarks on the Equivalence of Inertial and Gravitational Masses and on the Accuracy of Einstein's Theory of Gravity. Physics Essays, 18(2).

Hynecek, J. (2009a). New space-time metrics for symmetric spaces. Physics Essays, 22(2).

Hynecek, J. (2009). The Galileo effect and the general relativity theory. Physics Essays, 22(4).

\section{Appendix}

The metric derivation starts with the general form and the corresponding Lagrangian. For the simplicity of derivations the motion will be considered to occur only in the negative $z$ direction:

$$
\begin{gathered}
d s^{2}=g_{t t}(c d t)^{2}-g_{z z} d z^{2} \\
L=g_{t t}\left(\frac{c d t}{d \tau}\right)^{2}-g_{z z}\left(\frac{d z}{d \tau}\right)^{2}
\end{gathered}
$$

The first integral of Euler-Lagrange equation of motion for the time variable, derived similarly as in Equation 4, is as follows:

$$
d \tau=d t \cdot g_{t}
$$

For the test body that is stationary in the same uniform gravitational field $(d z / d \tau=0)$ and considering that the Lagrangian is also the first integral $\left(L=c^{2}\right)$ it follows that:

$$
d \tau=d t \sqrt{g_{t t}}
$$

Furthermore, considering that there must be a compatibility with the Special Relativity Theory where we have $g_{t t}=1$, the relation between the coordinate time $d t$ and the proper time $d \tau$ is as follows:

$$
d \tau=d t \sqrt{1-v^{2} / c^{2}}
$$

The general relations that satisfy all these conditions simultaneously are therefore as follows:

$$
\begin{gathered}
d \tau=d t \sqrt{g_{t t}} \sqrt{1-v^{2} / c^{2}} \\
g_{t t}=1-v^{2} / c^{2}
\end{gathered}
$$

From the Lagrangian $L=c^{2}$, substituting into it the relation from Equation A3, and including in the formula the definition of velocity $v=d z / d t$ results in the following:

$$
g_{t t}=1-\frac{g_{z z}}{g_{t t}} \frac{v^{2}}{c^{2}}
$$

Comparing now Equation A8 and Equation A7 it is clear that the following relation must hold:

$$
g_{z z}=g_{t t}
$$

The metric coefficients can now be found by applying the energy conservation rule between the energy of a body falling a certain distance thus attaining a corresponding velocity and the energy that is necessary for slowly lifting the body back to the same starting position. It is therefore clear that we must have for $z \leq 0$ the following relation:

$$
\frac{m_{0} c^{2}}{\sqrt{1-v^{2} / c^{2}}}=\frac{m_{0} c^{2}}{\sqrt{g_{t t}}}=m_{0} c^{2}+m_{0} g \cdot|z|
$$

From this result and Equation A7 that was used in Equation A10, follows that the metric coefficients are: 


$$
g_{t t}=g_{z z}=\frac{1}{\left(1-g \cdot z / c^{2}\right)^{2}}
$$

This finally leads to the metric given in Equation 1:

$$
d s^{2}=\frac{(c d t)^{2}}{\left(1-g \cdot z / c^{2}\right)^{2}}-\frac{d z^{2}}{\left(1-g \cdot z / c^{2}\right)^{2}}
$$

The complete and general derivation applicable also to various other symmetric spaces is available in the previous publication (Hynecek, 2009a).

The correctness of this metric is further justified by the fact that the speed of light in the $z$ direction is $c$, which validates the use of the standard energy formula of Equation 21 in Equation A10 and the fact that the force on the falling body is constant as derived in Equation 10, which validates the calculation of potential energy as given by Equation 13 .

\section{Copyrights}

Copyright for this article is retained by the author(s), with first publication rights granted to the journal.

This is an open-access article distributed under the terms and conditions of the Creative Commons Attribution license (http://creativecommons.org/licenses/by/4.0/). 